\title{
The identification and investigation of novel binding fragments in the Fatty Acid Binding Protein 6
}

\section{(FABP6).}

\author{
SUPPLEMENTARY INFORMATION
}

Alan G. Hendrick $t_{t}{ }^{*}$ Ilka Müller $t^{2}$ Henriëtte Willems, ${ }^{1}$ Philip M. Leonard, ${ }^{2}$ Steve Irving, ${ }^{3}$

Richard Davenport, ${ }^{1}$ Takashi Ito, ${ }^{4}$ Jenny Reeves, ${ }^{1}$ Susanne Wright,,${ }^{1}$ Vivienne Allen, ${ }^{2}$ Stephen

Wilkinson, ${ }^{2,}{ }^{\dagger}$ Helen Heffron, ${ }^{1}$ Richard Bazin, ${ }^{3}$ Jennifer Turney, ${ }^{2}$ and Philip J. Mitchell. ${ }^{1}$

${ }^{1}$ Takeda Cambridge, 418 Cambridge Science Park, Cambridge, CB40PZ, United Kingdom

${ }^{2}$ Charles River, Chesterford Research Park, Saffron Walden, Essex, CB10 1XL, United

Kingdom

${ }^{3}$ Charles River, Ingram Building, Parkwood Road, Canterbury, Kent, CT2 7NH, United

Kingdom

${ }^{4}$ Biomolecular Research Laboratories, Takeda Pharmaceutical Company Limited, 26-1, Muraoka-Higashi 2-chome, Fujisawa 251-8555, Japan

$\$$ These authors contributed equally. 


\section{CONTENTS}

Suppl. Figure 1: SPR Sensorgram for FABP6 binding to Compound 1.

Suppl. Figure 2: SPR Sensorgram for FABP1 binding to 8-Anilino-1-naphthalenesulfonic acid (ANS)

Suppl. Figure 3: Native mass spectrometry spectra for binding of Compound 1, cholate, GCDC, glycocholate and taurocholate to hFABP6. Competition study by native mass spectrometry between GCDC and Compound 1.

Suppl. Figure 4:Electron density maps for ligand binding site in apo-FABP6 structure.

Suppl. Table 1: $\mathrm{K}_{\mathrm{D}}$ values for FABP6 binding to bile salts

Suppl. Figure 1: (A) FABP6 SPR Sensorgram for Compound 1 with kinetic fit superposed (orange curve). Assuming a 1:1 binding event, the $\mathrm{K}_{\mathrm{D}}$ for Compound $\mathbf{1}$ is determined at $31 \mu \mathrm{M}$. (B) shows the steady state fit curve to the SPR data for Compound 1 for a 1:1 and 1:2 binding model, respectively. (C) FABP1 SPR Sensorgram for 8-Anilino-1-naphthalenesulfonic acid (ANS) with (D) a concentration-response curve showing a KD of $10.5 \mu \mathrm{M}$.

A

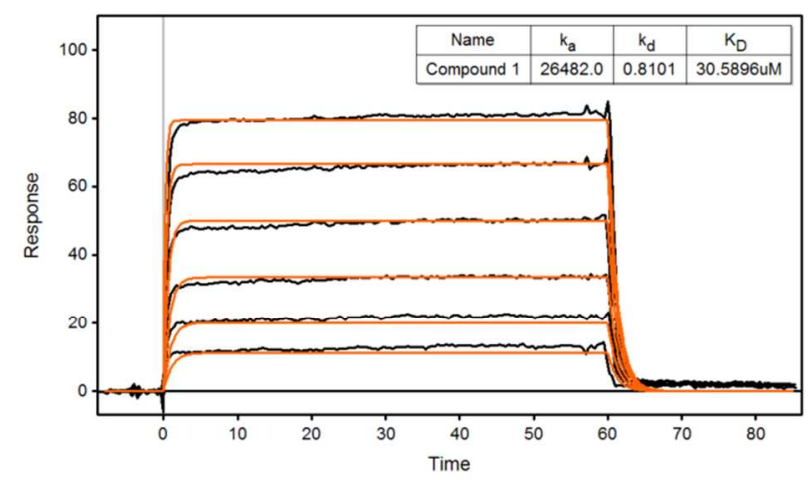


B
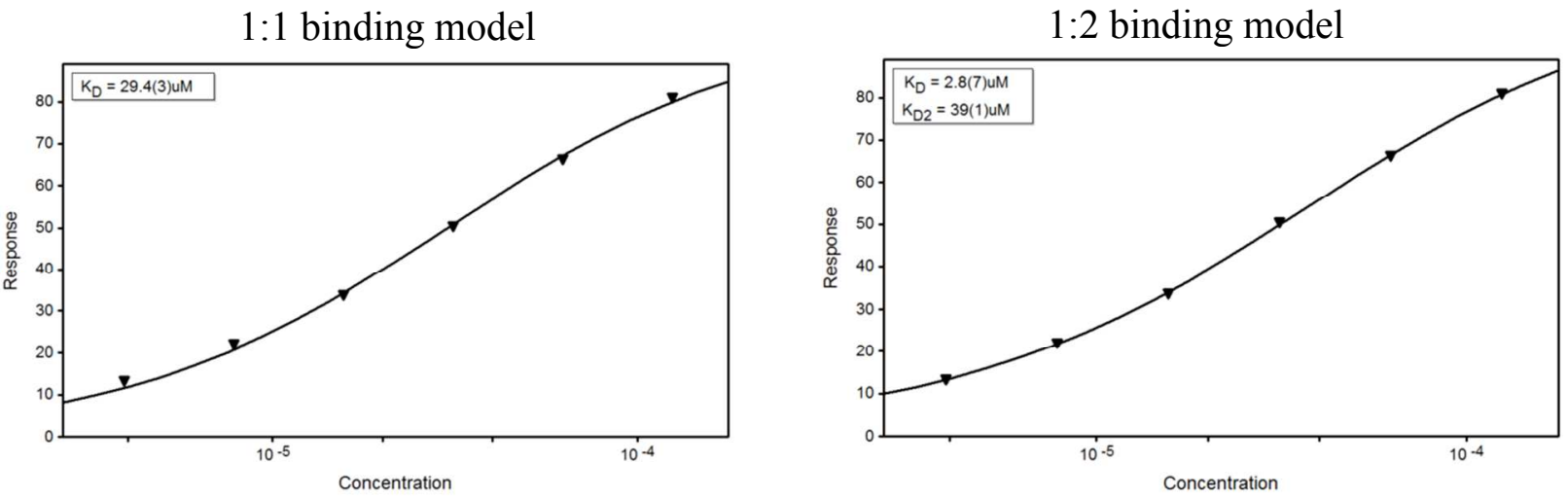

C

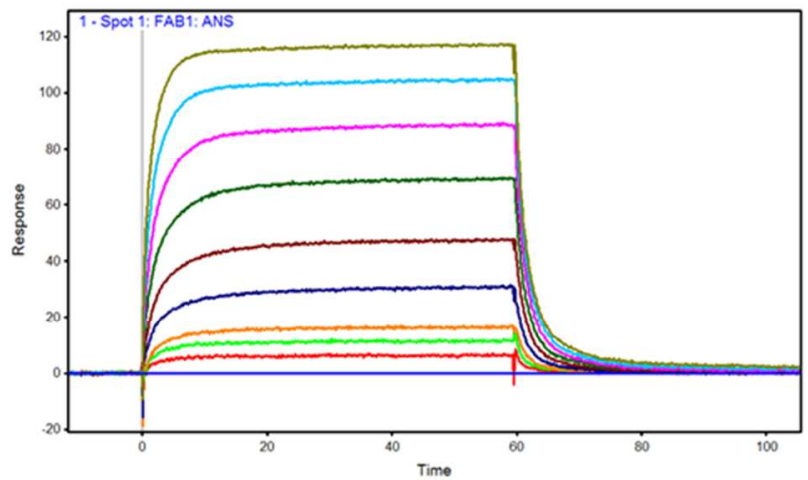

D

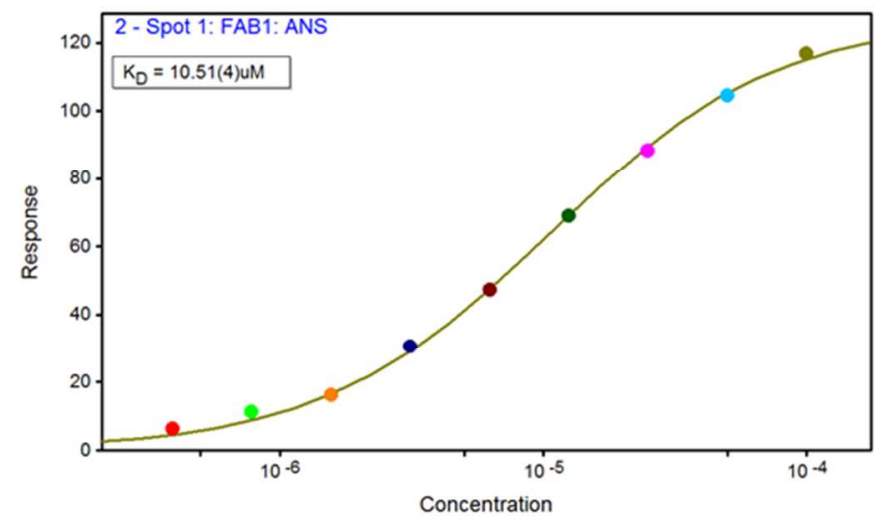


Suppl. Figure 2. Native mass spectrometry spectra for binding of Compound 1, cholate, GCDC, glycocholate and taurocholate to hFABP6 at a molecular ratio of protein to ligand of (A) $1: 5$ and (B) 1:10, respectively.

A

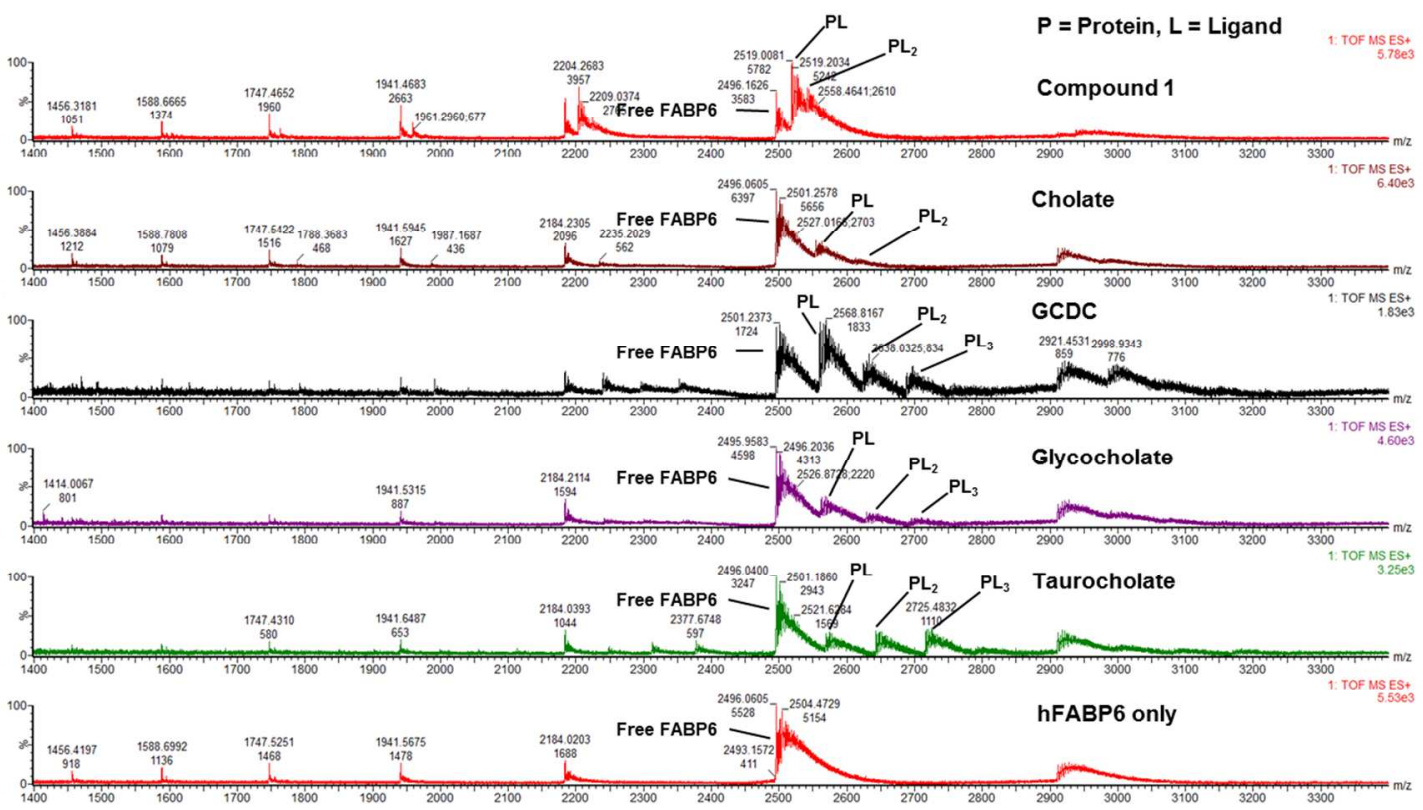

B

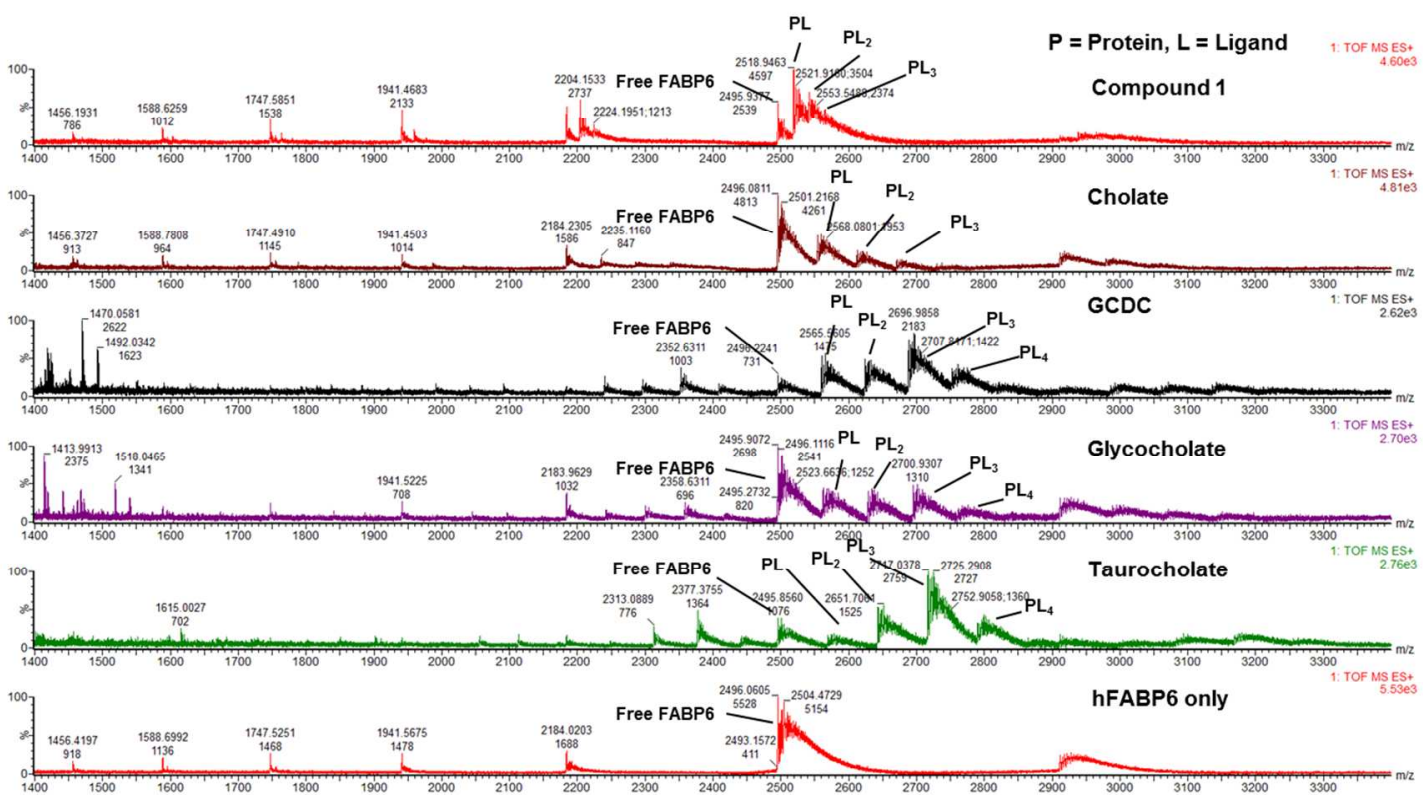


Suppl. Figure 3. Competition study by native mass spectrometry between GCDC and Compound 1. For the study, GCDC was used at 50 $\mu \mathrm{M}$, and hFABP6 at $10 \mu \mathrm{M}$.

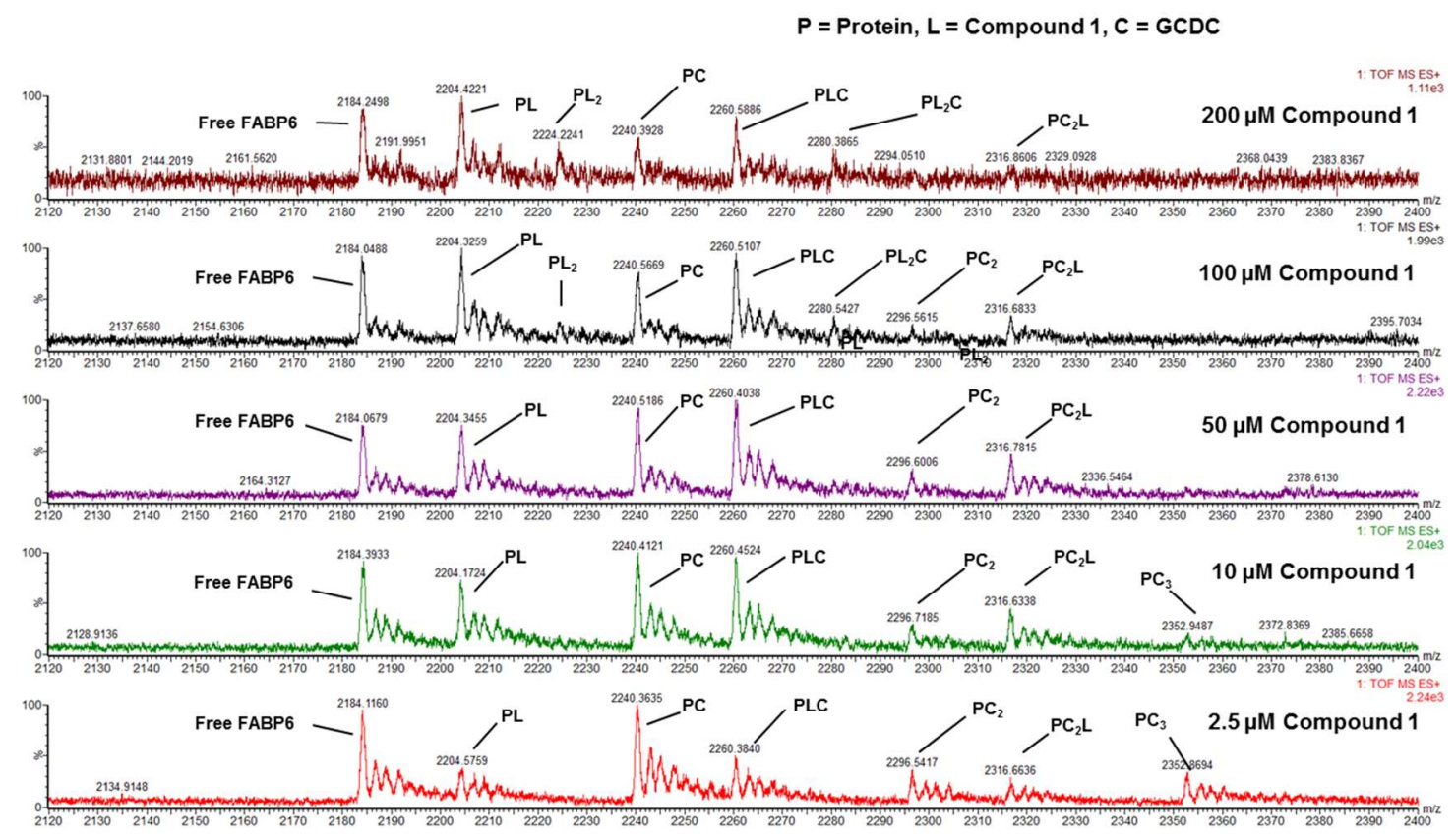

Suppl. Figure 4. Refined weighted 2Fo-Fc and Fo-Fc electron density maps of apo-hFABP6 around PEG binding sites in subunits A (left), B (center) and C (right). There is evidence of one PEG oligomer binding in all three subunits, and clear evidence of a second molecule binding in subunit B.

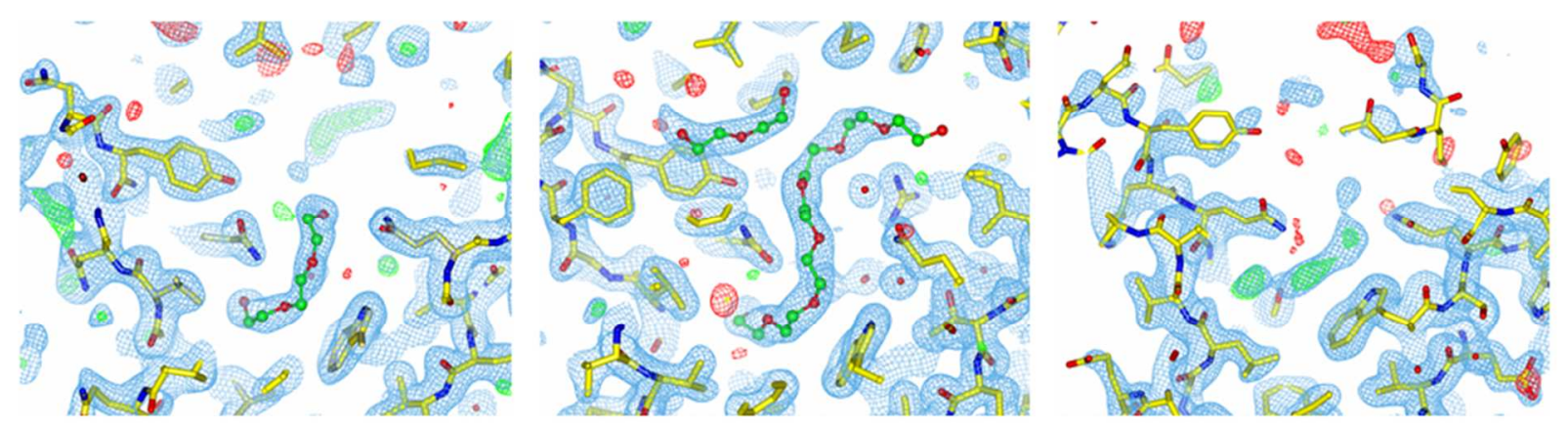


Suppl. Table 1: $K_{D}$ values for FABP6 binding to bile salts as assessed by SPR. Error values in parenthesis represent the standard deviation.

\begin{tabular}{|c|c|}
\hline Comp. & ${\text { FABP6 } \mathbf{K}_{\mathbf{D}}(\boldsymbol{\mu M})}$ \\
\hline Cholate & $415(5)$ \\
\hline Glycocholate & $595(6)$ \\
\hline Taurocholate & $95(5)$ \\
\hline Glycochenodeoxycholate & $200(1)$ \\
\hline
\end{tabular}

